\section{What is being studied as mindfulness meditation?}

\section{Megan S. Wheeler, Diane B. Arnkoff and Carol R. Glass}

In their recent Review (The neuroscience of mindfulness meditation. Nat. Rev. Neurosci. 16, 213-225 (2015) $)^{1}$, Tang, Hölzel and Posner present an admirable overview of the neuroscience of mindfulness. Their critique of cross-sectional studies raises an important point: practitioners could have "pre-existing differences ... which might be linked to their interest in meditation, personality or temperament." This highlights a notable confound in the neuroscience of mindfulness literature: research designs rarely distinguish intentional mindfulness meditation from a more intrinsic, dispositional mindfulness. Importantly, the latter has been found in the psychology literature to be protective against psychological distress ${ }^{2}-$ even for those without formal mindfulness training.

Disambiguating these two types of mindfulness will be important for bridging the gap between the neuroscientific and psychological literature, an integration that others have noted to be important ${ }^{3}$. Furthermore, decoupling the neural mechanisms underlying dispositional mindfulness from those underlying deliberate mindfulness will not only yield a more accurate view of the distinct neural correlates but also elucidate for whom mindfulness is most effective as a clinical intervention. This new knowledge could also facilitate new treatments aimed at improving wellbeing through increases in dispositional mindfulness in those unwilling to engage in formal mindfulness meditation.

Megan S. Wheeler, Diane B. Arnkoff and Carol R. Glass are at the Department of Psychology, O'Boyle Hall Room 314, The Catholic University of America, Washington DC 20064, USA.

Megan S. Wheeler is also at the Department of Psychological and Brain Sciences, 6207 Moore Hall, Hanover, New Hampshire 03755, USA.

Correspondence to M.S.W. megan.wheeler@dartmouth.edu

1. Tang, Y.-Y., Hölzel, B. K. \& Posner, M. I. The neuroscience of mindfulness meditation. Nat. Rev Neurosci. 16, 213-225 (2015).

2. Brown, K. W. \& Ryan, R. M. The benefits of being present: mindfulness and its role in psychological wellbeing. J. Pers. Soc. Psychol. 84, 822-848 (2003).

3. van der Velden, A. M. \& Roepstorff, A. Neural mechanisms of mindfulness meditation: bridging clinical and neuroscience investigations. Nat. Rev. Neurosci. 16, 439 (2015).

Competing interests statement The authors declare no competing interests 